PROCEEDINGS OF THE

AMERICAN MATHEMATICAL SOCIETY

Volume 134, Number 8, Pages 2271-2280

S 0002-9939(06)08235-9

Article electronically published on February 2, 2006

\title{
A FAMILY OF SCHOTTKY GROUPS ARISING FROM THE HYPERGEOMETRIC EQUATION
}

\author{
TAKASHI ICHIKAWA AND MASAAKI YOSHIDA \\ (Communicated by Carmen C. Chicone)
}

\begin{abstract}
We study a complex 3-dimensional family of classical Schottky groups of genus 2 as monodromy groups of the hypergeometric equation. We find non-trivial loops in the deformation space; these correspond to continuous integer-shifts of the parameters of the equation.
\end{abstract}

\section{INTRODUCTION}

When the three exponents of the hypergeometric differential equation are purely imaginary, its monodromy group is a classical Schottky group of genus 2; such groups form a real 3-dimensional family ([IY]). Since, under a small deformation, a Schottky group remains to be a Schottky group, by deforming the parameters of the hypergeometric equation, we have a complex 3-dimensional family of Schottky groups. We introduce (in $\S 4$ ) a complex 3-dimensional family $S$ of classical Schottky groups, containing the above ones coming from pure-imaginary-exponents cases, equipped with some additional structure. We study a structure of $S$, and construct loops (real 1-dimensional families of classical Schottky groups) in $S$ generating the fundamental group of $S$. These loops correspond to continous interger-shifts of the parameters of the hypergeometric equation.

Here we give a few examples of general background references. The hypergeometric function: [KSY], Schottky groups: Chapter 4 of [MSW] and Chapter 5 of BBEIM], history: Gray].

\section{The hyPERgeOMEtRIC EQUATION}

We consider the hypergeometric differential equation

$$
E(a, b, c): x(1-x) \frac{d^{2} u}{d x^{2}}+\{c-(a+b+1) x\} \frac{d u}{d x}-a b u=0 .
$$

For (any) two linearly independent solutions $u_{1}$ and $u_{2}$, the (multi-valued) map

$$
s: X:=\mathbb{C}-\{0,1\} \ni x \longmapsto z=u_{1}(x): u_{2}(x) \in \mathbb{P}^{1}:=\mathbb{C} \cup\{\infty\}
$$

is called a Schwarz map (or Schwarz's $s$-map). If we choose as solutions $u_{1}$ and $u_{2}$, near $x=0$, a holomorphic one and $x^{1-c}$ times a holomorphic one, respectively,

Received by the editors October 1, 2004 and, in revised form, February 24, 2005.

2000 Mathematics Subject Classification. Primary 33C05, 30F10, 30F40.

Key words and phrases. Hypergeometric equation, monodromy group, Schottky group.

(C)2006 American Mathematical Society 
then the circuit matrices around $x=0$ and 1 are given by

$$
\gamma_{1}=\left(\begin{array}{cc}
1 & 0 \\
0 & e^{2 \pi i(1-c)}
\end{array}\right) \quad \text { and } \quad \gamma_{2}=P^{-1}\left(\begin{array}{cc}
1 & 0 \\
0 & e^{2 \pi i(c-a-b)}
\end{array}\right) P,
$$

respectively, where $P$ is a connection matrix given by

$$
P=\left(\begin{array}{cc}
\frac{\Gamma(c) \Gamma(c-a-b)}{\Gamma(c-a) \Gamma(c-b)} & \frac{\Gamma(2-c) \Gamma(c-a-b)}{\Gamma(1-a) \Gamma(1-b)} \\
\frac{\Gamma(c) \Gamma(a+b-c)}{\Gamma(a) \Gamma(b)} & \frac{\Gamma(2-c) \Gamma(a+b-c)}{\Gamma(a-c+1) \Gamma(b-c+1)}
\end{array}\right) ;
$$

here $\Gamma$ denotes the Gamma function. Note that the matrices act on the row vector $\left(u_{1}, u_{2}\right)$ from the right. These generate the monodromy group $M(a, b, c)$ of the equation $E(a, b, c)$.

\section{Hypergeometric equations With PURELy imaginary EXPONENT-DIFFERENCES ([SY], [Y] $)$}

If the exponent-differences

$$
\lambda=1-c, \quad \mu=c-a-b, \quad \nu=b-a
$$

(at the singular points $x=0,1$ and $\infty$, respectively) are purely imaginary, the image of the upper-half part

$$
X^{+}:=\{x \in X \mid \Im x \geq 0\}
$$

is bounded by the three circles (in the $s$-plane) which are images of the three intervals $(-\infty, 0),(0,1)$ and $(1,+\infty)$; put

$$
C_{1}=s((-\infty, 0)), \quad C_{3}=s((0,1)), \quad C_{2}=s((0,+\infty)) .
$$

Note that if we continue $s$ analytically through the interval, say $(0,1)$, to the lowerhalf part $X^{-}:=\{x \in X \mid \Im x \leq 0\}$, then the image of $X^{-}$is the mirror image of that of $X^{+}$under the reflection with center $C_{3}$.

The reflection with center (mirror) $C_{j}$ will be denoted by $\rho_{j}(j=1,2,3)$. The monodromy group $M(a, b, c)$ is the group consisting of even words of $\rho_{j}(j=1,2,3)$. This group is a Schottky group of genus 2 . The domain of discontinuity modulo $M(a, b, c)$ is a curve of genus 2 defined over the reals.

Choosing the solutions $u_{1}$ and $u_{2}$ suitably, we can assume that the centers of the three circles are on the real axis, and that the circles $C_{2}$ and $C_{3}$ are inside the circle $C_{1}$ (see Figure 1). Let $C_{1}^{\prime}$ and $C_{2}^{\prime}$ be the mirror images with respect to $C_{3}$ of the circles $C_{1}$ and $C_{2}$, respectively. Then $\gamma_{1}:=\rho_{3} \rho_{1}$ maps the inside of $C_{1}$ onto the inside of $C_{1}^{\prime}$; and $\gamma_{2}:=\rho_{3} \rho_{2}$ maps the inside of $C_{2}$ onto the outside of $C_{2}^{\prime}$. Note that one of the two fixed points of $\gamma_{1}$ is inside $C_{1}^{\prime}$, and the other one is outside $C_{1}$; one of the two fixed points of $\gamma_{2}$ is inside $C_{2}^{\prime}$, and the other one is inside $C_{2}$. The transformations $\gamma_{1}$ and $\gamma_{2}$ are conjugate to those given in $\S 2$.

We thus have four disjoint disks $D_{1}, D_{1}^{\prime}, D_{2}$, and $D_{2}^{\prime}$, whose centers are on the real axis, and two loxodromic transformations $\gamma_{1}$ and $\gamma_{2}$ taking $D_{1}$ and $D_{2}$ to the complementary disks of $D_{1}^{\prime}$ and $D_{2}^{\prime}$, respectively. Note that one of the two fixed points of $\gamma_{1}$ is in $D_{1}^{\prime}$ and the other one in $D_{1}$; one of the two fixed points of $\gamma_{2}$ is in $D_{2}^{\prime}$ and the other one in $D_{2}$. 

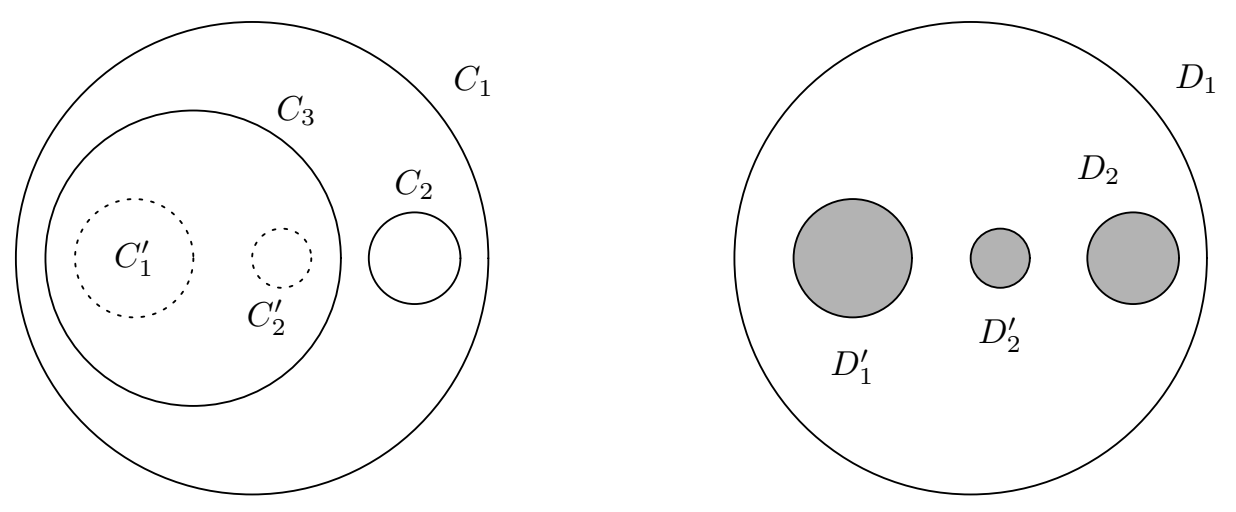

FiguRE 1. The circles and the disks

\section{THE MOdUli SPACE $S$}

Two loxodromic transformations $\gamma_{1}$ and $\gamma_{2}$ generate a (classical) Schottky group if and only if there are four disjoint closed disks $D_{1}, D_{1}^{\prime}, D_{2}$, and $D_{2}^{\prime}$, such that $\gamma_{1}$ and $\gamma_{2}$ take $D_{1}$ and $D_{2}$ to the complementary disks of $D_{1}^{\prime}$ and $D_{2}^{\prime}$, respectively. Throughout the paper, disks are always assumed to be closed; we simply call them disks. The closure of the complement of a disk in $\mathbb{P}^{1}$ will simply be called the complementary disk of the disk.

Definition. Let $S$ be the space of two loxodromic transformations $\gamma_{1}$ and $\gamma_{2}$ equipped with four disjoint disks $D_{1}, D_{1}^{\prime}, D_{2}$, and $D_{2}^{\prime}$, such that $\gamma_{1}$ and $\gamma_{2}$ take $D_{1}$ and $D_{2}$ to the complementary disks of $D_{1}^{\prime}$ and $D_{2}^{\prime}$, respectively.

The space has a natural structure of a complex 3-dimensional manifold. We are interested in its homotopic property.

Note that if a loxodromic transformation $\gamma$ takes a disk $D$ onto the complementary disk of a disk $D^{\prime}\left(D \cap D^{\prime}=\emptyset\right)$, then $\gamma$ has a fixed point in $D$ and the other fixed point in $D^{\prime}$.

A loxodromic transformation is determined by the two fixed points and the multiplier.

Lemma 1. For two given disjoint disks $D$ and $D^{\prime}$, there is a unique point $F \in D$ (resp. $F^{\prime} \in D^{\prime}$ ) such that by a(ny) linear fractional transformation taking $F$ (resp. $\left.F^{\prime}\right)$ to $\infty$, the two circles $\partial D$ and $\partial D^{\prime}$ are transformed into concentric circles.

Proof. Let the two disks be given as

$$
D:|z-a| \leq r, \quad D^{\prime}:\left|z-a^{\prime}\right| \leq r^{\prime} .
$$

By the transformation $z \rightarrow w$ defined by

$$
z=\frac{1}{w}+\zeta
$$

taking $\zeta$ to $\infty$, the circles $C=\partial D$ and $C^{\prime}=\partial D^{\prime}$ are transformed into circles with centers

$$
c:=\frac{\bar{\zeta}-\bar{a}}{r^{2}-|\zeta-a|^{2}}, \quad c^{\prime}:=\frac{\bar{\zeta}-\bar{a}^{\prime}}{r^{\prime 2}-\left|\zeta-a^{\prime}\right|^{2}}
$$


respectively. Equating $c$ and $c^{\prime}$, we have the quadric equation

$$
\zeta^{2}+\left(-a-a^{\prime}+\frac{r^{2}-r^{\prime 2}}{\bar{a}-\bar{a}^{\prime}}\right) \zeta+a a^{\prime}+\frac{a r^{\prime 2}-a^{\prime} r^{2}}{\bar{a}-\bar{a}^{\prime}}=0 .
$$

Put $\zeta=\left(a-a^{\prime}\right) \eta+a^{\prime}$. Note that $\zeta=a^{\prime}$ and $a$ correspond to $\eta=0$ and 1 , respectively. Then the equation above for $\zeta$ reduces to

$$
\eta^{2}+\left(-1+\frac{r^{2}-r^{\prime 2}}{\left|a-a^{\prime}\right|^{2}}\right) \eta+\frac{r^{\prime 2}}{\left|a-a^{\prime}\right|^{2}}=0 .
$$

It is a high school mathematics problem to see that each of the two roots of this equation is in each of the two intervals

$$
\left(0, \frac{r^{\prime}}{\left|a-a^{\prime}\right|}\right) \text { and }\left(1-\frac{r}{\left|a-a^{\prime}\right|}, 1\right) .
$$

Since concentric circles are mapped to concentric circles under linear transformations, this completes the proof.

Remark 1. The circles $\partial D$ and $\partial D^{\prime}$ are Apollonius circles with respect to the two centers $F$ and $F^{\prime}$.

Lemma 2. For two given disjoint disks $D$ and $D^{\prime}$ and a point $f^{\prime}$ in the interior of $D^{\prime}$, there is a 1-parameter family (parametrized by a circle) of loxodromic transformations $\gamma$ which take $D$ onto the complementary disk of $D^{\prime}$, and fix $f^{\prime}$. The absolute value $|m|$ of the multiplier $m$ of $\gamma$ is determined by the given data.

(1) If $f^{\prime} \neq F^{\prime}$, then the other fixed point $f \in D$ of $\gamma$ must be on the Apollonius circle $A=A\left(f^{\prime}\right)$ in $D$ determined by the two centers of the circles $\partial D$ and $\partial D^{\prime}$, and proportion $|m|$. $\arg m \in \mathbb{R} / 2 \pi \mathbb{Z}$ determines $f$, and vice versa.

(2) If $f^{\prime}=F^{\prime}$, then the other fixed point is $F \in D$. $\arg m \in \mathbb{R} / 2 \pi \mathbb{Z}$ remains free.

Proof. We can assume that $f=0$ and $f^{\prime}=\infty$, so that the transformation in question can be presented by $z \mapsto m z$. Let $c$ and $r$ be the center and the radius of the disk $D$, and $c^{\prime}$ and $r^{\prime}$ those of the complementary disk of $D^{\prime}$. Then we have $c^{\prime}=m c$ and $r^{\prime}=|m| r$.

(1) If $c \neq c^{\prime}, f(=0)$ is on the Apollonius circle

$$
A:\left|f-c^{\prime}\right|=|m||f-c|
$$

with centers $c$ and $c^{\prime}$, and proportion $|m|$. It is easy to see that this circle is in $D$. (See Figure 2.)

(2) If $c=c^{\prime}=0$, then the Apollonius circle reduces to a point.

In case (2), we blow up the point $F$ to be the circle $\mathbb{R} / 2 \pi \mathbb{Z}$, and call this circle also the Apollonius circle $A$. Under this convention, two disjoint disks $D$ and $D^{\prime}$, an interior point $f^{\prime} \in D^{\prime}$, and a point $f$ on the Apollonius circle $A$ uniquely determine a loxodromic transformation.

Thus an element of $S$ can be determined by four disjoint disks $D_{1}, D_{1}^{\prime}, D_{2}, D_{2}^{\prime}$, two interior points $f_{1}^{\prime} \in D_{1}^{\prime}, f_{2}^{\prime} \in D_{2}^{\prime}$, and two points $f_{1}$ on the Apollonius circle $A_{1}$ (determined by $D_{1}, D_{1}^{\prime}, f_{1}^{\prime}$ ) in $D_{1}$, and $f_{2}$ on the Apollonius circle $A_{2}$ (determined by $\left.D_{2}, D_{2}^{\prime}, f_{2}^{\prime}\right)$ in $D_{2}$. Since disks are contractible, we have

Proposition 1. The fundamental group of $S$ can be generated by the moves of the four disjoint disks $D_{1}, D_{1}^{\prime}, D_{2}, D_{2}^{\prime}$, and the moves of $f_{1}$ in $A_{1}$, and $f_{2}$ in $A_{2}$. 


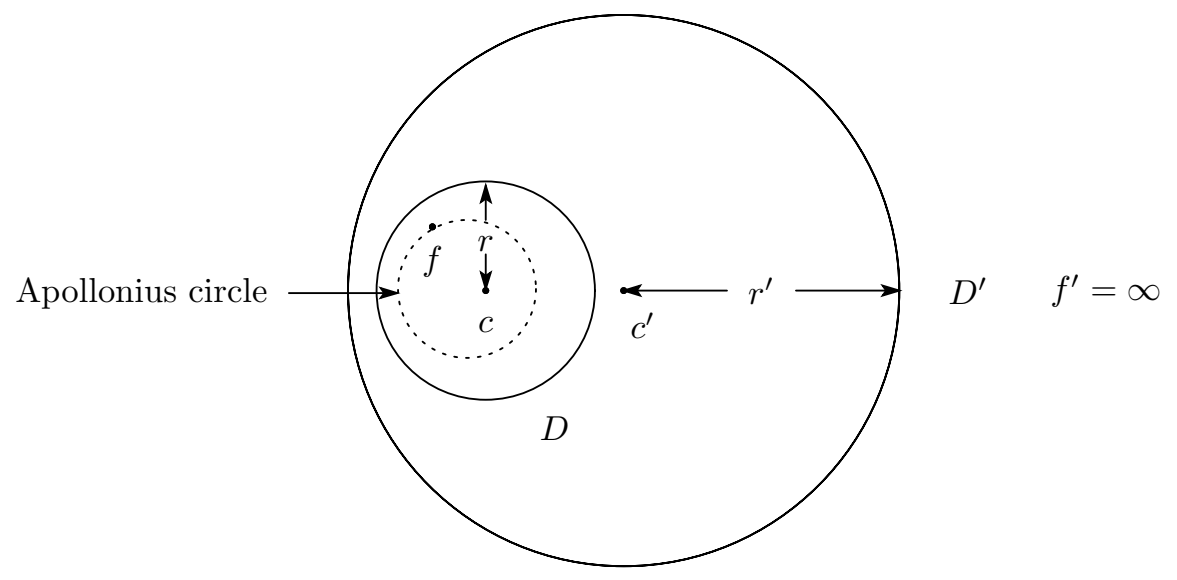

Figure 2. Apollonius circle in $D$

In the next section we explicitly construct loops (real 1-dimensional families of classical Schottky groups) in $S$.

\section{LOOPS IN $S$}

Let $\gamma_{1}$ and $\gamma_{2}$ be as in $\S 2$. The fixed points of $\gamma_{1}$ are $\{0, \infty\}$, and those of $\gamma_{2}$ are

$$
f_{2}=\frac{\Gamma(c) \Gamma(a-c+1) \Gamma(b-c+1)}{\Gamma(2-c) \Gamma(a) \Gamma(b)} \quad \text { and } \quad f_{2}^{\prime}=\frac{\Gamma(c) \Gamma(1-a) \Gamma(1-b)}{\Gamma(2-c) \Gamma(c-a) \Gamma(c-b)} .
$$

We change the coordinate $z$ by multiplying by $1 / f_{2}$. Then the fixed points of $\gamma_{1}$ remain to be $\{0, \infty\}$, and those of $\gamma_{2}$ become 1 and

$$
\alpha=g(a) g(b), \quad \text { where } \quad g(x)=\frac{\sin (\pi c-\pi x)}{\sin (\pi x)} .
$$

Indeed we have

$\frac{f_{2}^{\prime}}{f_{2}}=\frac{\Gamma(1-a) \Gamma(1-b)}{\Gamma(c-a) \Gamma(c-b)} \cdot \frac{\Gamma(a) \Gamma(b)}{\Gamma(a-c+1) \Gamma(b-c+1)} \quad$ and $\quad \Gamma(x) \Gamma(1-x)=\frac{\pi}{\sin (\pi x)}$.

Definition. When the three exponent-differences are purely imaginary:

$$
\lambda=i \theta_{0}, \quad \mu=i \theta_{1}, \quad \nu=i \theta_{2}, \quad \theta_{0}, \theta_{1}, \theta_{2}>0,
$$

the generators $\left\{\gamma_{1}, \gamma_{2}\right\}$ of the monodromy group of $E(a, b, c)$ given in $\S 2$ (take the four disks given in $\S 3$ ) form a simply connected real 3 -dimensional submanifold of $S$; this submanifold is called $S_{0}$.

In this section, we construct loops in $S$, with base in $S_{0}$, which generate the fundamental group of $S$. When the exponent-differences are as above, note that the parameters can be expressed as

$$
a=\frac{1}{2}-\frac{i}{2}\left(\theta_{0}+\theta_{1}+\theta_{2}\right), \quad b=\frac{1}{2}-\frac{i}{2}\left(\theta_{0}+\theta_{1}-\theta_{2}\right), \quad c=1-i \theta_{0} .
$$

For clarity, we denote the four disks $D_{1}, D_{1}^{\prime}, D_{2}, D_{2}^{\prime}$ by

$$
D_{0}(\ni 0), \quad D_{\infty}(\ni \infty), \quad D_{1}(\ni 1), \quad D_{\alpha}(\ni \alpha) .
$$



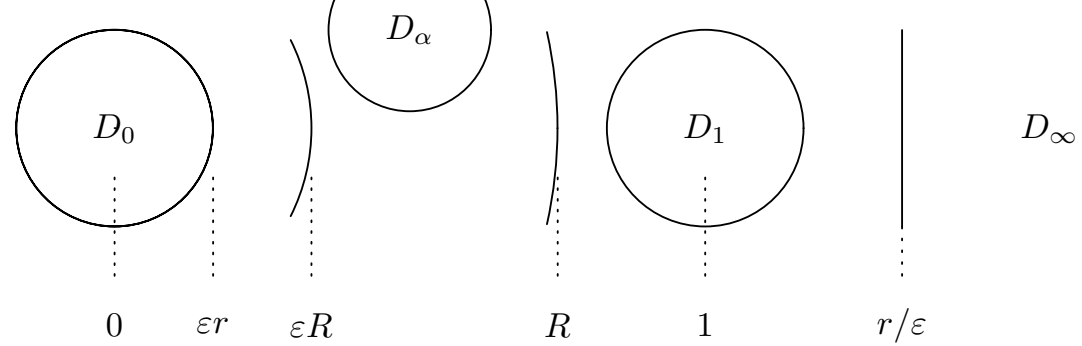

Figure 3 . The disk $D_{\alpha}$ travels around the disk $D_{0}$

5.1. The disk $D_{\alpha}$ travels around the disk $D_{0}$. We construct a loop in $S$ (with base in $S_{0}$ ), which is represented by a travel of the disk $D_{\alpha}$ around the disk $D_{0}$ with a change of argument (by $2 \pi$ ) of the multiplier of $\gamma_{2}$. We fix $c$ and the real part of $a$ (as $1 / 2)$, and let the real part of $b$ move from $1 / 2$ to $3 / 2$; the imaginary parts of $a$ and $b$ are so chosen that the monodromy group $M(a, b, c)$ remains to be a Schottky group along the move. Putting $c=1-i \theta_{0}$, we have

$$
g(x)=\frac{\varepsilon^{-1}-\varepsilon e^{2 \pi i x}}{1-e^{2 \pi i x}}=\varepsilon+\frac{\varepsilon^{-1}-\varepsilon}{1-e^{2 \pi i x}}, \quad \text { where } \quad \varepsilon=e^{-\pi \theta_{0}}<1 .
$$

Set

$$
r=g\left(\frac{1}{2}-\frac{i}{2}\left(\theta_{0}+2\right)\right), \quad R=g\left(\frac{1}{2}-\frac{i}{2}\left(\theta_{0}+1\right)\right) .
$$

Then we have

$$
0<\varepsilon<r=\varepsilon \frac{e^{2 \pi}+\varepsilon^{-1}}{e^{2 \pi}+\varepsilon}<R=\varepsilon \frac{e^{\pi}+\varepsilon^{-1}}{e^{\pi}+\varepsilon}<1 .
$$

Let $D_{0}$ be the disk with center at 0 and with radius $\varepsilon r$, and $D_{\infty}$ the complementary disk of the disk with center at 0 and with radius $\varepsilon^{-1} r$. Note that we have

$$
0<\varepsilon r<\varepsilon R<R<1<\varepsilon^{-1} r,
$$

and that $\gamma_{1}$ maps $D_{0}$ onto the outside disk of $D_{\infty}$.

Define real continuous functions $\phi(t)$ and $\psi(t)$ for $0 \leq t \leq 1$ by

$$
R e^{2 \pi i t}=g\left(\frac{1}{2}+\phi(t)-\frac{i}{2}\left(\theta_{0}+\psi(t)\right)\right) .
$$

Since

$$
2 \pi i\left(\frac{1}{2}+\phi(t)-\frac{i}{2}\left(\theta_{0}+\psi(t)\right)\right)=\log \left(R e^{2 \pi i t}-\varepsilon^{-1}\right)-\log \left(R e^{2 \pi i t}-\varepsilon\right),
$$

the function $\phi(t)$ is monotone increasing with $\phi(0)=0, \phi(1)=1$, and $\psi(t)$ satisfies $\psi(0)=\psi(1)=1$. Choose $\theta_{1}$ so large that

- $\theta_{1}>\max \{\psi(t) \mid 0 \leq t \leq 1\}$, and that

- for any $z$ in the ring $\{\varepsilon R \leq|z| \leq R\}$, there are two disjoint disks $D_{1}$ ( $\ni 1$ ) and $D_{z}(\ni z)$, and a fractional linear transformation with multiplier $e^{-2 \pi \theta_{1}}$ mapping $D_{1}$ onto the complementary disk of $D_{z}$. 
Now set $\theta_{2}(t)=\theta_{1}-\psi(t)>0$ and deform the parameters $a$ and $b$ as

$$
\begin{aligned}
& a(t)=\frac{1}{2}-\frac{i}{2}\left(\theta_{0}+\theta_{1}+\theta_{2}(t)\right), \\
& b(t)=\frac{1}{2}+\phi(t)-\frac{i}{2}\left(\theta_{0}+\theta_{1}-\theta_{2}(t)\right) .
\end{aligned}
$$

Then we have $\varepsilon<g(a(t))<1,|g(b(t))|=R$, and so $\alpha(t)=g(a(t)) g(b(t))$ satisfies

$$
\varepsilon R<|\alpha(t)|=g(a(t))|g(b(t))|<R \text {. }
$$

Thus $\alpha(t)$ travels around the disk $D_{0}$ in the ring $\{\varepsilon R \leq|z| \leq R\}$, and there are two disjoint disks $D_{1}(\ni 1)$ and $D_{\alpha(t)}(\ni \alpha)$ in the ring $\left\{\varepsilon r<|z|<\varepsilon^{-1} r\right\}$, and a transformation $\gamma_{2}(t)$ with multiplier $e^{2 \pi i \mu}=e^{-2 \pi \theta_{1}}$ which maps $D_{1}$ onto the complementary disk of $D_{\alpha(t)}$.

5.2. The disk $D_{\alpha}$ travels around the disk $D_{1}$. We construct a loop in $S$ (with base in $S_{0}$ ), which is represented by a travel of the disk $D_{\alpha}$ around the disk $D_{1}$ with a change of argument (by $2 \pi$ ) of the multiplier of $\gamma_{2}$. We fix $c, a$ and the imaginary part of $b$, and let the real part of $b$ move from $1 / 2$ to $3 / 2$. Take $\theta_{0}>0$ and $\theta^{\prime}<0$ satisfying

$$
\frac{e^{\pi \theta^{\prime}}\left(1+e^{\pi \theta^{\prime}}\right)}{1-e^{\pi \theta^{\prime}}}<\varepsilon^{-2}, \quad \varepsilon=e^{-\pi \theta_{0}}
$$

Set

Since we have

$$
r=\varepsilon+\frac{\varepsilon^{-1}-\varepsilon}{1+e^{\pi \theta^{\prime}}}, \quad R=\varepsilon+\frac{\varepsilon^{-1}-\varepsilon}{1-e^{\pi \theta^{\prime}}} .
$$

$$
\frac{1-\varepsilon r}{r}=\frac{1-\varepsilon^{2}}{r}\left(1-\frac{1}{1+e^{\pi \theta^{\prime}}}\right)>0
$$

and

$$
\varepsilon^{-1} r-\varepsilon R=\left(1-\varepsilon^{2}\right)\left(1+\frac{\varepsilon^{-2}}{1+e^{\pi \theta^{\prime}}}-\frac{1}{1-e^{\pi \theta^{\prime}}}\right)>0,
$$

where the last inequality holds thanks to (1), there is a positive number $s$ satisfying

$$
0<s<\min \left\{r, \frac{1-\varepsilon r}{r}, \frac{\varepsilon^{-1} r-\varepsilon R}{\varepsilon^{-1}+R}\right\},
$$

which implies

$$
(\varepsilon+s) r<1, \quad(\varepsilon+s) R<\varepsilon^{-1}(r-s) .
$$

Take $\theta_{1}$ so large that

$$
\varepsilon+\frac{\varepsilon^{-1}-\varepsilon}{1+e^{\pi\left(\theta_{0}+\theta_{1}\right)}}<\varepsilon+s .
$$

Now set $\theta_{2}:=\theta_{0}+\theta_{1}-\theta^{\prime}$ and

$$
a=\frac{1}{2}-\frac{i}{2}\left(\theta_{0}+\theta_{1}+\theta_{2}\right)
$$

and deform (the real part of) $b$ as

Note that

$$
b(t)=\frac{1}{2}+t-\frac{i}{2}\left(\theta_{0}+\theta_{1}-\theta_{2}\right)=\frac{1}{2}+t-\frac{i}{2} \theta^{\prime} .
$$

$$
\varepsilon<g(a)=\varepsilon+\frac{\varepsilon^{-1}-\varepsilon}{1+e^{\pi\left(\theta_{0}+\theta_{1}+\theta_{2}\right)}}<\varepsilon+s \quad \text { (here we used (3)) }
$$



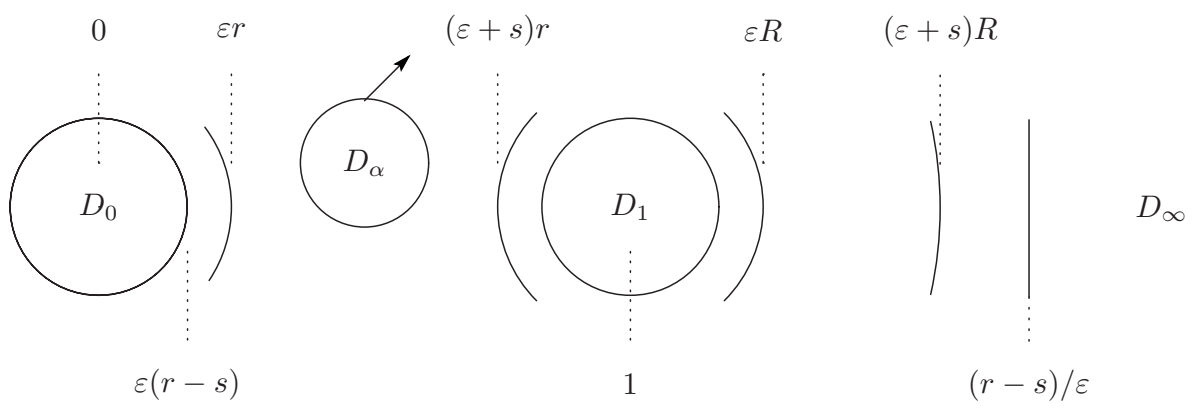

Figure 4 . The disk $D_{\alpha}$ travels around the disk $D_{1}$

and that

$$
g(b(t))=\varepsilon+\frac{\varepsilon^{-1}-\varepsilon}{1+e^{\pi \theta^{\prime}} e^{2 \pi i t}} \quad \text { satisfies } \quad r \leq|g(b(t))| \leq R .
$$

These inequalities together with (2) imply that $\alpha(t)=g(a) g(b(t))$ is in the ring $\{\varepsilon r \leq|z| \leq(\varepsilon+s) R\}$, and that

$$
\alpha(0)=\alpha(1) \leq(\varepsilon+s) r<1<\varepsilon R \leq \alpha(1 / 2) .
$$

This shows that $\alpha(t)$ travels around 1 . Let $D_{0}$ be the disk with center at 0 and with radius $\varepsilon(r-s), D_{\infty}$ the complementary disk of the disk with center at 0 and with radius $\varepsilon^{-1}(r-s)$. Then $\gamma_{1}$ maps $D_{0}$ onto the complementary disk of $D_{\infty}$, and by the right side of (2), $D_{0} \cup D_{\infty}$ is disjoint from the ring

$$
\{\varepsilon r \leq|z| \leq(\varepsilon+s) R\} \ni 1, \alpha(t) .
$$

Thus by taking $\theta_{1}>0$ sufficiently large, for any $0 \leq t \leq 1$, there are two disjoint disks $D_{1}(\ni 1), D_{\alpha(t)}(\ni \alpha(t))$ in the complement of $D_{0} \cup D_{\infty}$ and a transformation $\gamma_{2}(t)$ with multiplier $e^{2 \pi i \mu}=e^{-2 \pi \theta_{1}} e^{-2 \pi i t}$ which maps $D_{1}$ onto the complementary disk of the $D_{\alpha(t)}$ (see Figure 4 ).

5.3. The multiplier of $\gamma_{2}$ travels around 0 . We construct a loop in $S$ (with base in $S_{0}$ ), which is represented by the change of argument (by $2 \pi$ ) of the multiplier of $\gamma_{2}$. We fix $b$ and $c$, and the imaginary part of $a$, and let the real part of $a$ move from $1 / 2$ to $3 / 2$. Set

$$
\theta:=\theta_{0}+\theta_{1}+\theta_{2}, \quad \varepsilon:=e^{-\pi \theta_{0}}, \quad r:=\frac{\varepsilon^{-1}-\varepsilon}{e^{\pi \theta}-1} .
$$

Choose and fix $\theta_{0}, \theta_{1}$ and $\theta_{2}$ so that $\theta_{1}=\theta_{2}$ and

$$
r<\min \left\{1-\varepsilon, \frac{1}{1+\varepsilon^{-1}}\right\} \text {. }
$$

Then since

$$
b=\frac{1}{2}-\frac{i}{2}\left(\theta_{0}+\theta_{1}-\theta_{2}\right)=\frac{1}{2}-\frac{i}{2} \theta_{0},
$$

we have $e^{2 \pi i b}=-\varepsilon^{-1}$, and so $g(b)=1$.

Now we deform the parameter $a$ as

$$
a(t)=\frac{1}{2}+t-\frac{i}{2} \theta, \quad 0 \leq t \leq 1 .
$$




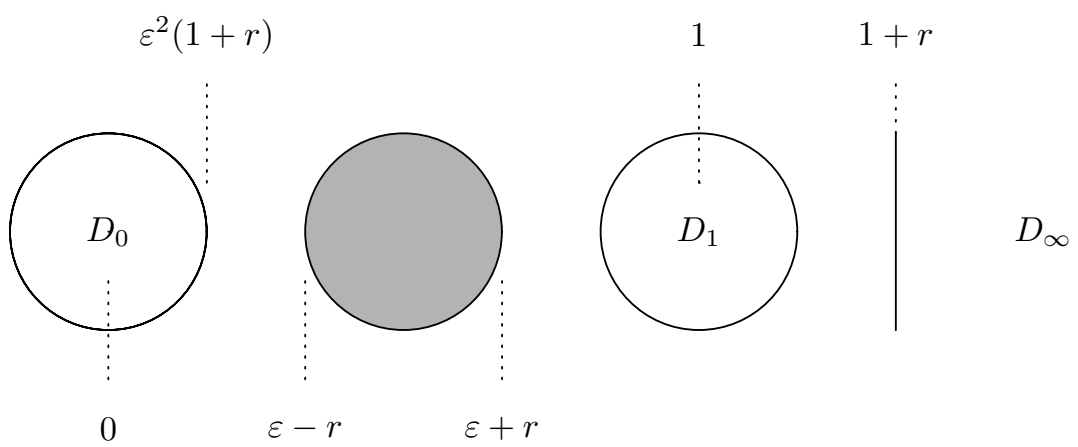

Figure 5. The fixed point $\alpha$ of $\gamma_{2}$ travels along the Apollonius circle

Since we have

$$
\alpha(t)=g(a(t)) g(b)=g(a(t))=\varepsilon+\frac{\varepsilon^{-1}-\varepsilon}{1+e^{\pi \theta} e^{2 \pi i t}},
$$

the point $\alpha$ is in the disk with center at $\varepsilon$ and with radius $r$. We thus have

$$
\varepsilon^{2}(1+r)<\varepsilon-r \leq|\alpha(t)| \leq \varepsilon+r<1<1+r .
$$

Let $D_{0}$ be the disk with center at 0 and with radius $\varepsilon^{2}(1+r), D_{\infty}$ the complementary disk of the disk with center at 0 and with radius $1+r, D_{\alpha(t)}(\ni \alpha(t))$ the disk with center at $\varepsilon$ and with radius $r$. Then $\gamma_{1}$ maps $D_{0}$ onto the complementary disk of $D_{\infty}$, and $\alpha(t)$ belongs to the disk $\{|z-\varepsilon| \leq r\}$ which is disjoint from $D_{0} \cup D_{\infty} \cup\{1\}$. Thus by taking $\theta_{1}=\theta_{2}>0$ sufficiently large, for any $0 \leq t \leq 1$, there are two disjoint disks $D_{1}(\ni 1), D_{\alpha(t)}(\ni \alpha(t))$ in the complement of $D_{0} \cup D_{\infty}$ and a transformation $\gamma_{2}(t)$ with multiplier $e^{2 \pi i \mu}=e^{-2 \pi \theta_{1}} e^{-2 \pi i t}$ which maps $D_{1}$ onto the complementary disk of $D_{\alpha(t)}$ (see Figure 5).

5.4. The multiplier of $\gamma_{1}$ travels around 0 . We construct a loop in $S$ (with base in $S_{0}$ ), which is represented by the change of argument (by $2 \pi$ ) of the multiplier of $\gamma_{1}$. Note that by the change of variable $x \rightarrow 1-x$, the equation $E(a, b, c)$ changes into $E(a, b, a+b+1-c)$. So we have only to literally follow $\S 5.3$ exchanging $c$ and $a+b+1-c$, and $\theta_{0}$ and $\theta_{1}$. We thus fix $b$ and the imaginary parts of $a$ and $c$, and let the real part of $c$ move from 1 to 2 , and let the real part of $a$ move from $1 / 2$ to $3 / 2$ keeping $a+b+1-c$ constant.

\section{MiscellaneA}

For a Schottky group $\Gamma$ of genus 2, the quotient of the domain of discontinuity modulo $\Gamma$ is a curve of genus 2. A curve of genus 2 is a double cover of $\mathbb{P}^{1}$ branching at six points, which are uniquely determined modulo automorphisms of $\mathbb{P}^{1}$ by the curve. Thus a Schottky group determines a point of the configuration space $X\{6\}$ of six-point sets on $\mathbb{P}^{1}$. (The fundamental group of $X\{6\}$ is the Braid group with five strings.) When all the exponent-differences of the hypergeometric equation are purely imaginary, its monodromy group is a Schottky group, which determines six points on a line. Thus they determine a point of the configuration space $X(6)$ of six colored points on $\mathbb{P}^{1}$. (The fundamental group of $X(6)$ is the colored Braid group with five strings.) The space $X(6)$ is well studied. 
Remark 2. When all the exponent-differences are purely imaginary, there is a Schottky automorphic function defined by an absolutely convergent infinite product, which induces a holomorphic map of the genus 2 curve onto $\mathbb{P}^{1}([\mathrm{IY}])$. This infinite product remains to be convergent for groups represented by loops above if we take the multipliers of $\gamma_{1}$ and $\gamma_{2}$ sufficiently small. This is because there is a circle separating two disks among the four (BBEIM, Chapter 5]).

Problems. The four loops constructed in $\S 5$ induce those in $X\{6\}$. Do they generate the fundamental group of $X\{6\}$ ? Same problem for $X(6)$.

\section{ACKNOWLEDGEMENTS}

The authors are grateful to Professors Susumu Hirose, Ken'ichi Ohshika, Hiroki Sato and Hiro-o Yamamoto for their encouragement.

\section{REFERENCES}

[BBeiM] E. D. Belokolos, A. I. Bobenko, V.Z. Enol'skit, A. R. Its and V. B. Matveev, Algebro-geometric Approach to Nonlinear Integrable Equations, Springer Series in Nonlinear Dynamics (Springer-Verlag, 1994).

[GP] L. Gerritzen and M. van der Put, Schottky groups and Mumford curves, Lect. Notes in Math. 817(1980) Springer. MR0590243 (82j:10053)

[IY] T. ICHIKAWA AND M. YoshidA, On Schottky groups arising from the hypergeometric equation with imaginary exponents, Proc AMS 132(2003), 447-454. MR2022368

[IKSY] K. Iwasaki, H. Kimura, S. Shimomura And M. Yoshida, From Gauss to Painlevé A modern theory of special functions, Vieweg Verlag, Wiesbaden, 1991. MR1118604 (92j:33001)

[Gray] J. GRAY, Linear differential equations and group theory from Riemann to Poincaré, Birkhäuser, 2000. MR:1751835 (2000m:34002)

[MSW] D. Mumford, C. Series And D. Wright, Indra's Pearls, Cambridge Univ. Press, 2002. MR:1913879(2003f:00005)

[SY] T. SASAKI AND M. YOSHIDA, A geometric study of the hypergeometric function with imaginary exponents, Experimental Math. 10(2000), 321-330. MR1917420 (2003f:33002)

[Sch] F. SсноттKY, Über eine specielle Function, welche bei einer bestimmten linearen Transformation ihres Arguments univerändert bleibt, J. Reine Angew. Math. 101 (1887) 227272 .

Department of Mathematics, Faculty of Science and Engineering, Saga University, SAGA 840-8502, JAPAN

E-mail address: ichikawa@ms.saga-u.ac.jp

Department of Mathematics, Kyushu University, Fukuoka 810-8560, Japan

E-mail address: myoshida@math.kyushu-u.ac.jp 